\title{
CD63 and C3ARI: The Potential Molecular Targets in the Progression of Septic Shock
}

\author{
Ning Yu, Xuefang Liu, Dandan Shi, Long Bai, Tianfu Niu, Ya Liu \\ Department of Anaesthesiology and Intensive Care, The Second Hospital of Hebei Medical University, Shijiazhuang, Hebei Province, 050004, People's
} Republic of China

Correspondence: Ya Liu, Department of Anaesthesiology and Intensive Care, The Second Hospital of Hebei Medical University, Shijiazhuang, Hebei Province, 050004, People's Republic of China, Email md2006liuya@aliyun.com; liuya6660707@I63.com

Background: The molecular mechanism of septic shock is unknown. We studied the pathogenesis of septic shock and provide a novel strategy for treating and improving the prognosis of septic shock.

Methods: Gluten-Sensitive Enteropathy (GSE) 131761, GSE119217, GSE26378 datasets were downloaded from the Gene Expression Omnibus (GEO) database. The three datasets included 204 septic shock samples and 48 normal samples. The R packages "affy" and "limma" were employed to identify the differently expressed genes (DEGs) between septic shock and normal samples. Weighted gene co-expression network analysis (WGCNA) was performed to search for modules that play an important role in septic shock. Functional annotation of DEGs and construction and analysis of hub genes were used to explore the pathomechanism of septic shock. The receiver operating characteristic (ROC) curves were obtained using MedCalc software. The drug molecules that could regulate hub genes associated with septic shock were searched for in the CMap database. An animal model of septic shock was constructed to analyze the role of these hub genes.

Results: The merged series contained 321 up-regulated and 255 down-regulated genes. WGCNA showed the brown module had the highest correlation with the status of septic shock. GO and KEGG enrichment analysis results of the brown module genes showed they were mainly enriched in "leukocyte differentiation", "Ras-proximate-1 (Rap1) signaling pathway", and "cytokine-cytokine receptor interaction". Through construction and analysis of a protein-protein interaction (PPI) network, cluster of differentiation 63 (CD63) and complement component 3a receptor 1 (C3AR1) were identified as hub genes of septic shock. The area under curve (AUC) of C3AR1 for the septic shock is $0.772(\mathrm{P}<0.001)$, and the AUC of CD63 for the septic shock is $0.871(\mathrm{P}<0.001)$. Small molecule drugs were filtered by the number of instances $(\mathrm{n}>3)$ and P-values $<0.05$, including "monensin", "verteporfin", "ikarugamycin", "tetrahydroalstonine", "cefamandole", "etoposide". In the animal model, the relative expression levels of interleukin-6 (IL-6), Tumor Necrosis Factor- $\alpha(\mathrm{TNF}-\alpha)$, and lactic acid were significantly higher in the septic shock group compared with the control group. Results of Real Time Quantitative PCR (RT-qPCR) and enzyme-linked immunosorbent assay (ELISA) analysis for CD63 and C3AR1 showed that their relative expression levels were significantly lower in the septic shock group compared with the control group ( $\mathrm{P}<0.05)$.

Conclusion: CD63 and C3AR1 are significant hub genes of septic shock and may represent potential molecular targets for future studies of septic shock.

Keywords: septic shock, CD63, C3AR1, hub genes, animal model

\section{Introduction}

Sepsis is defined as a life-threatening organ dysfunction caused by a dysregulated host response to infection. ${ }^{1}$ This systemic inflammatory response syndrome can be caused by infections that arise as a serious complication in patients with acute clinical conditions, such as trauma, burn, shock, infection, and major surgery; it is also an important cause of septic shock and multiple organ dysfunction syndrome (MODS). ${ }^{2}$ Septic shock can be clinically diagnosed as persistent hypotension despite adequate fluid resuscitation, requiring vasopressor to maintain mean arterial pressure greater than $65 \mathrm{~mm} \mathrm{Hg}$, and serum lactic acid elevation greater than $2 \mathrm{mmol} / \mathrm{L} \cdot{ }^{3-5}$ Recent studies have shown that the number of patients with sepsis in China has reached more than 4 million each year. Data from the United States show that there are 1.7 million cases of sepsis there each year, with a case fatality rate of $28.6 \%$. Although modern emergency medicine has 
made great progress, the incidence of sepsis continues to increase year by year. ${ }^{6}$ When sepsis is associated with multiple organ dysfunction, mortality is significantly increased. ${ }^{7,8}$ Therefore, the study of the pathogenesis of septic shock, its early identification, timely diagnosis, and appropriate intervention are key to effectively preventing the occurrence and development of septic shock and improving its treatment success rates.

Recent studies have found that during the development of septic shock, the excessive release of inflammatory mediators causes the body's immune system to over-react and coagulation dysfunction to occur, which are key to determining the severity and prognosis of septic shock. ${ }^{9}$ In septic shock, various endogenous inflammatory mediators, including vasoactive substances, cytokines, acute-phase reactive substances, plasma enzyme system products, and fibrinolytic pathways, interact to form network effects and cause extensive damage to various body systems. ${ }^{10}$ Platelets also play an important role in the complex interaction between inflammation and blood clotting. ${ }^{11}$ However, the molecular mechanism of septic shock remains unclear.

Bioinformatics has become an important research frontier in the field of biomedicine. ${ }^{12}$ In fact, with the rapid development of high-throughput sequencing technology, biomedical data is now facing the era of biomedical big data. ${ }^{12}$ Genome sequencing has become a necessary and essential tool, with genomics playing a central role in providing new insights into various aspects of biology to further unravel difficult questions about human health and disease. ${ }^{13-15}$

This study used bioinformatics technology to reveal the core genes involved in septic shock and to carry out enrichment analysis, pathway analysis, and survival analysis. The potential roles of the core genes identified in septic shock were validated using an animal model.

\section{Materials and Methods}

Data from the GEO Database

The GEO database is a database that stores chips, second-generation sequencing and other high-throughput sequencing data. Using this database, we can retrieve some experimental sequencing data uploaded by some other people. We obtained the transcriptome expression profiles GSE131761 (GPL13497), GSE119217 (GPL16686), and GSE26378 (GPL570) from the GEO database (Table 1). The three datasets included 204 septic shock samples and 48 normal samples, which were used to identify DEGs between the septic shock and normal groups. The principle of integration or merger analysis for the data was followed. First, the three datasets had the uniform gene symbol. Second, the sample size was expanded via the merger analysis. Third, the expressed data is normalized via the sva package under $\mathrm{R}$ language. The sva package could remove the effect of batch effect and other irrelevant variables in high-throughput experiments. There are two steps: First, identify and evaluate potential influence variables in the experiment; Second, directly apply ComBat to remove known batch effects.

\section{Screening of DEGs}

The three GEO series were merged and normalized using Perl. The R packages "affy" and "limma" were employed to perform probe summarization and background correction of GSE131761, GSE119217, and GSE26378. The BenjaminiHochberg method was used to adjust the original p-values. Fold-changes (FC) were calculated using the false discovery rate (FDR). The cut-off criteria for DEGs were $|\log \mathrm{FC}|>1$ and $\mathrm{p}$-value $<0.05$.

Table I A Summary of Sepsis Shock Microarray Dataset from Different GEO Datasets

\begin{tabular}{|l|l|l|l|c|c|}
\hline & Series & Platform & Affymetrix GeneChip & Normal & Sepsis Shock \\
\hline I & GSEI3I76I & GPLI3497 & $\begin{array}{l}\text { Agilent-026652 Whole Human Genome } \\
\text { Microarray 4x44K v2 (Probe Name version) }\end{array}$ & 15 & 81 \\
\hline 2 & GSEI192I7 & GPLI6686 & $\begin{array}{l}\text { [HuGene-2_0-st] Affymetrix Human Gene } \\
2.0 \text { ST Array [transcript (gene) version] }\end{array}$ & 12 & $4 I$ \\
\hline 3 & GSE26378 & GPL570 & $\begin{array}{l}\text { [HG-UI33_Plus_2] Affymetrix Human } \\
\text { Genome UI33 Plus 2.0 Array }\end{array}$ & 21 & 82 \\
\hline
\end{tabular}




\section{Weighted Gene Co-Expression Network Analysis (WGCNA)}

WGCNA is a bioinformatics analysis method used to describe gene association patterns between different samples. Genes with similar expression patterns can be clustered and the association between modules and specific traits or phenotypes can be analyzed. The WGCNA of all genes in the merged series was performed using the R package "WGCNA" (https://cran.r-project.org/web/packages/WGCNA/index.html). A co-expression network for all genes was constructed, and the algorithm filtered the $25 \%$ of genes with the largest variation for further analysis. Then, 204 septic shock samples and 48 normal samples were used for the WGCNA analysis. An adjacency matrix was created for the samples, which was then transformed into a topological overlap matrix (TOM). Genes were divided into different gene modules using TOM-based difference measurement. The minimal gene module was $>50$ and the threshold to merge similar modules was 0.1 ; these values were then used to search for modules that play an important role in septic shock.

\section{Functional Annotation of DEGs}

The GO and KEGG analyses are computational methods that evaluate gene functions and biological pathways. Gene Set Enrichment Analysis (GSEA) (http://software.broadinstitute.org/gsea/index.jsp) is a computational method that can execute GO and KEGG analysis with a given gene list. The Metascape (http://metascape.org/gp/index.html) database provides a comprehensive gene annotation and analysis resource. In this study, GO and KEGG analyses of target genes were performed to explore the potential mechanism by which module genes promote the progression of septic shock.

\section{Construction and Analysis of the PPI Network}

Funrich (http://www.funrich.org/) is biological analysis software. FunRich is a stand-alone software tool used mainly for functional enrichment and interaction network analysis of genes and proteins. The DEGs in the brown model genes were analyzed using Funrich. STRING (http://string-db.org), an online database, can be used to predict and provide the PPI network after importing the brown model DEGs. Cytoscape is an analysis tool that can provide biological network analysis and two-dimensional (2D) visualization for biologists. In our study, the PPI network was constructed and analyzed using the STRING database and Cytoscape, and the hub DEGs were screened using four algorithms. Venn diagrams were then used to show the intersection between the four groups of hub DEGs to obtain common hub DEGs using Funrich.

\section{Identification of Hub DEGs Associated with Septic Shock}

The comparative toxicogenomics database (CTD, http://ctdbase.org/) is a web-based database. It provides manually curated information about chemical-gene/protein interactions, chemical-disease and gene-disease relationships. These data are integrated with functional and pathway data to aid in development of hypotheses about the mechanisms underlying environmentally influenced diseases. The relationship between genes/proteins and disease can be predicted using the CTD. In our study, the relationships between genes products and septic shock were analyzed using this database.

\section{Functional Annotation}

DIANA-Path is a RNA pathway analysis web-server, providing accurate statistics, while being able to accommodate advanced pipelines. DIANA-Path v3.0 is an online analysis tool suite dedicated to conducting functional and pathway enrichment analyses. In our study, GO and KEGG pathway enrichment analyses were performed $(P<0.05)$.

\section{ROC Analysis}

ROC curves are drawn according to a series of different dichotomies (cut-off value or determination threshold) with the true positive rate (sensitivity) as the ordinate and the false positive rate (1-specificity) as the abscissa. The receiver operating characteristic (ROC) curves were obtained using MedCalc software. 


\section{Identification of Candidate Molecule Drugs for Hub Genes}

The Connectivity Map (also known as cmap) is a collection of genome-wide transcriptional expression data from cultured human cells treated with bioactive small molecules. The connectivity map (CMap, https://portals.broadinstitute. org/cmap/) database can connect genes and genomic information with human diseases and drugs used to treat them. In this study, the drug molecules that could regulate hub genes associated with septic shock were searched for in the CMap database.

\section{Animal Model Method}

The C57 black 6 (C57BL/6) mice (Male, $8 \pm 1$ Weeks) were weighed, and this information was recorded. They were then numbered and assigned to groups according to the random number table method. Anesthetize the mouse by injecting intraperitoneally a solution of $1: 1 \mathrm{ketamine}(75 \mathrm{mg} / \mathrm{kg}$, Shanghai Civic Chemical Technology Co., Ltd, Shanghai) and xylazine $(15 \mathrm{mg} / \mathrm{kg})$. As a reference, inject $30 \mu \mathrm{L}$ of the $1: 1$ solution into a mouse weighing 20 grams. Alternatively, mice can be anesthetized with inhaled isoflurane using an anesthetic vaporizer. The mice were fixed in a supine position on the operating table following successful anesthesia. Routine skin preparation was performed in the central abdominal area. A sterile perforated towel was used for routine abdominal disinfection. An incision about 1-cm-long was made along the linea alba of the abdomen using a scalpel. The cortex, muscular, and peritoneal layers were divided, layer by layer. Following laparotomy, the mesentery and cecum were dissociated, and one third of the junction of the ileum and cecum was ligated with 4-0 silk thread along the root of cecum, to avoid damage to the surrounding mesenteric vessels. At 8$12 \mathrm{~mm}$ from the distal end of the ligation site, an $18 \mathrm{G}$ puncture needle was used to penetrate the intestinal wall twice, perpendicular to the long axis of the cecum, and an appropriate amount of intestinal content was extruded. The cecum is returned to the peritoneal cavity and the peritoneum is closed with 6.0 silk sutures. The skin is closed with Reflex $7 \mathrm{~mm}$ clips (RS-9258, Roboz Surgical Instruments) or Michel wound clips (7 mm, RS-9270). Provide free access to food and water (hydrogel) placed on the bottom of the cage. ${ }^{16}$ In the sham operation group, the abdomens were closed after turning the cecum, while in the normal operation group, no operation was performed.

This study was approved by the Ethics Committee of the second hospital of Hebei Medical University. Written informed consent was obtained from all patients. All experiments were approved by Animal Care and Use Committee of the Hebei Medical University. All institutional and national guidelines for the care and use of laboratory animals were followed.

\section{ELISA}

ELISA has become a leading topic in the field of analytical chemistry. It is a special reagent analysis method. ELISA involves binding an antibody to an enzyme complex and detecting it with color. An ELISA is often used to detect large molecular antigens in the peripheral blood; the general steps were performed as follows. The specific antibody was coated on a plastic pore plate, and any excess antibody was washed off after completion. The sample was added. If the antigen to be measured is contained in the sample, it will bond specifically with the antibody on the plastic disk. Superfluous sample was washed away, and another antigen-specific primary antibody was added to bond with the antigen to be tested. The excess unbonded primary antibody was washed away, and a secondary antibody with enzyme was added to bond with the primary antibody. The excess unbonded secondary antibodies were washed away, the enzyme substrate was added to color the enzyme, and the result of the color was read using the naked eye or an appropriate instrument.

The main reagents used by the experiment: Mouse IL-6 ELISA kit (ab222503, Abcam, Cambridge, UK), Mouse tumor necrosis factor (TNF) alpha ELISA kit (ab 208348, Abcam, Cambridge, UK), Mouse lactic acid ELISA kit (Shanghai Keshun Biological Technology Co., Ltd, Shanghai, China), Mouse CD63 ELISA Kit (LSBio, LS-F6927, USA), Mouse C3AR1 ELISA kit (Abbexa, abx392273, UK).ELISA to use serum for detection and centrifugation to prepare serum: (1) After the blood is extracted, it can be immediately put into the centrifugal tube and quickly covered, placed vertically on the centrifuge test tube rack, and transferred to a low greenhouse. (2) Prepare to centrifuge blood cells and serum in a low greenhouse. (3) Place the centrifuge tube containing blood symmetrically in the centrifuge sample chamber and cover the chamber well. (4) Set centrifugal parameters: The general speed can be set at $4000 \mathrm{rpm}$, 
and the centrifugal time can be set for 5 minutes. (5) Start the machine for centrifugal separation, power off after the centrifugation, open the sample bin, carefully take out the sample, observe the sample separation, if not ideal, centrifugal again, until satisfied. You'll notice that the blood cells are tightly packed at the bottom of the tube, and there's a lot of serum in the tube. (6) Carefully siphon the serum from the top layer with a pipette. Store it in a refrigerated storage container and freeze it immediately.

\section{Polymerase Chain Reaction (PCR)}

Polymerase chain reaction (PCR) is a molecular biological technology used to amplify and amplify specific DNA fragments. It can be regarded as special DNA replication in vitro. The biggest characteristic of PCR is that it can greatly increase the amount of DNA. The extraction of total RibonucleicAcid (RNA) from the peripheral blood of septic shock mice was performed according to the total RNA extraction kit (B0004DP, Shanghai Haifang Biotechnology Co., LTD) instructions. The purity and concentration of the total RNA was determined. Reverse transcription of complementary Deoxyribonucleic acid (cDNA) was then performed. DNA was denatured at $90^{\circ} \mathrm{C}$ to $96^{\circ} \mathrm{C}$. Under the action of heat, the hydrogen bonds of the double-stranded DNA template breaks, forming single-stranded DNA. Annealing was carried out at $60^{\circ} \mathrm{C}$ to $65^{\circ} \mathrm{C}$. As the temperature of the system decreased, the primer bound to the DNA template to form a local double strand. In the extension step, at $70^{\circ} \mathrm{C}$ to $75^{\circ} \mathrm{C}$, and under the action of Thermus aquaticus (Taq) enzyme using deoxy-ribonucleoside triphosphate (dNTP) as the raw material, a DNA molecule complementary to the template is synthesized from the 3' end of the primer and extending in a 5' to 3' direction. Following denaturation, annealing, and extension, the DNA content doubles with each cycle. Primers of CD63, C3AR1 and their sequences for PCR analysis were followed:

CD63-F: GGCTGAGGGAATGAGAATTAAGGCA,

CD63-R: TGCCTTAATTCTCATTCCCTCAGCC.

C3AR1-F: GTCCTCAACATGTTTGCCAGTGTCT.

C3AR1-R: AGACACTGGCAAACATGTTGAGGAC.

The specificity of PCR primers for CD63 was $100 \%$, and the C3AR1 was $87 \%$.

\section{Results}

\section{Identification of DEGs}

The merged series contained 321 up-regulated and 255 down-regulated genes (Figure 1A). A heat map of DEG expression is shown in Figure 1B.

\section{WGCNA Results}

In our study, WGCNA was conducted using the R package "WGCNA". The selection of the soft-thresholding power was an important step in the WGCNA. Network topology analysis was performed to identify the soft-thresholding power. The soft-thresholding power in the WGCNA was set to 6, which was the lowest power for the scale-free topology fit index of 0.9 (Figure 1C). A hierarchical clustering tree of all genes was constructed, and 15 important modules were generated (Figure 1D). The dendrograms and heatmaps of genes showed that there was no significant difference in interactions among the different modules, demonstrating that there was a high degree of independence between them (Figure 1E). The brown module had the highest correlation with the status of septic shock (Figure 1F). Interactions between these modules were then analyzed (Figure 1G).

\section{Functional and Pathway Enrichment Analysis of DEGs}

The enrichment results of GO and KEGG analysis of DEGs, performed by GSEA, showed they were mainly enriched in "positive regulation of interleukin 1 production" and "peroxisome proliferator-activated receptor (PPAR) signaling pathway" (Figure 2A and B). The enrichment results of GO and KEGG analysis of brown model genes performed by Metascape showed they were mainly enriched in "leukocyte differentiation", "Rap1 signaling pathway", "cytokinecytokine receptor interaction", and others (Figure 2C-F). 
A

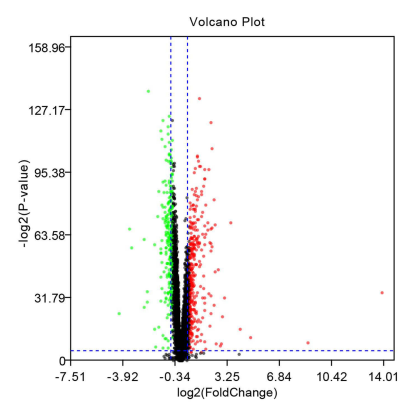

C

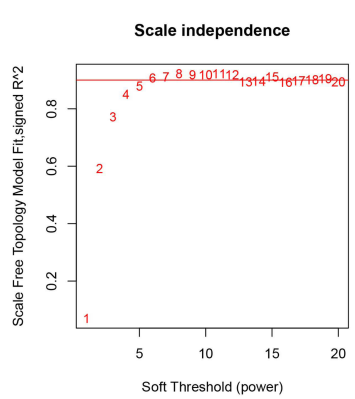

B

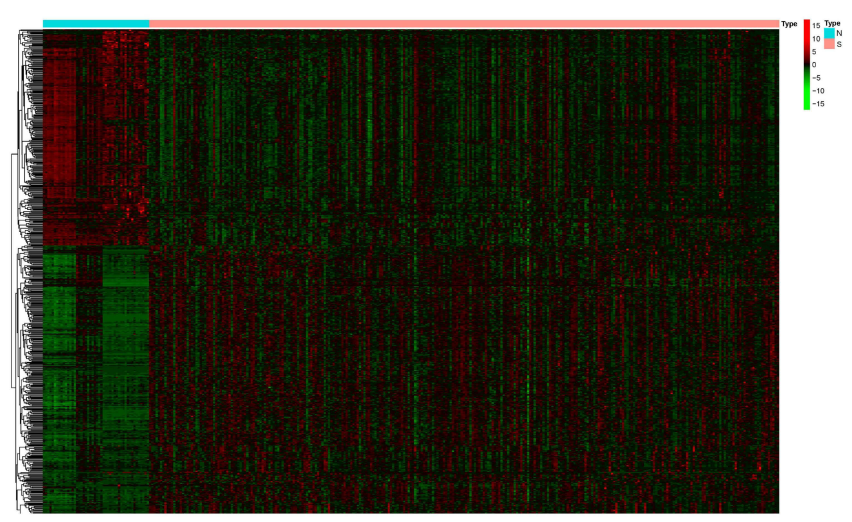

D

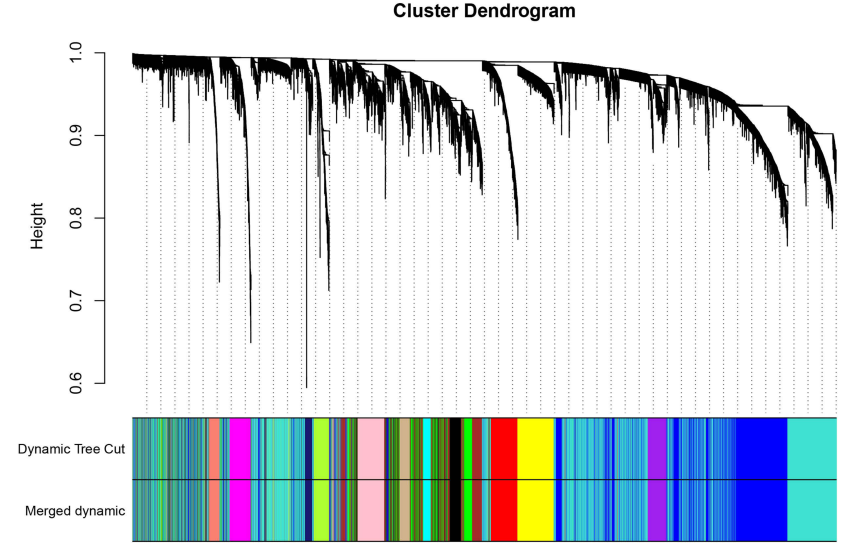

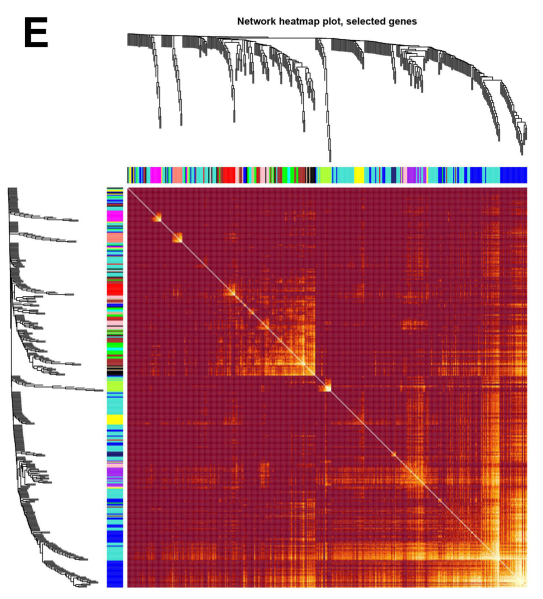

F

G
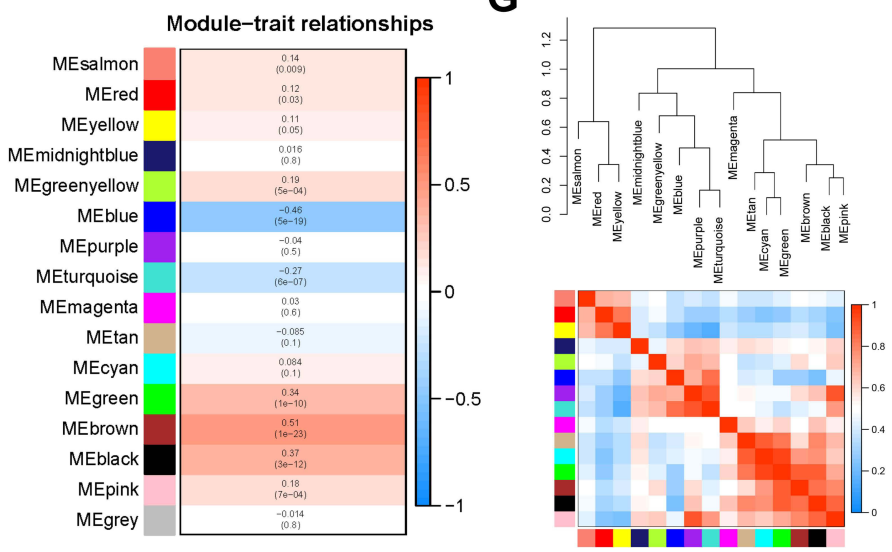

Figure I Differential expression analysis and weighted gene co-expression network analysis (WGCNA) of the genes in the merged series. (A) Volcano plots of the genes showing differentially expressed Genes (DEGs) between the septic shock and normal groups. (B) Heatmaps of the 576 DEGs. (C) The lowest power for which scale Independence was observed. (D) Repeated hierarchical clustering tree of all genes. (E) The dendrogram and heatmap of all genes. (F) Interactions between these modules. (G) The associations between clinical traits and the modules.

\section{Construction and Analysis of the PPI Network}

A Venn diagram is used to show the intersection between the module genes and DEGs (Figure 3A). The PPI network of DEGs in the brown module was constructed using the STRING online database and analyzed by Cytoscape software (Figure 3B). Four different algorithms were employed to identify hub genes, and four common hub genes were obtained (Figure 3C). A summary of the common hub genes is shown in Table 2. The PPI network of common hub genes is shown in Figure 3D.

\section{Identification of Hub Genes}

The CTD database shows common hub genes associated with septic shock, as shown in Figure 4. 
A

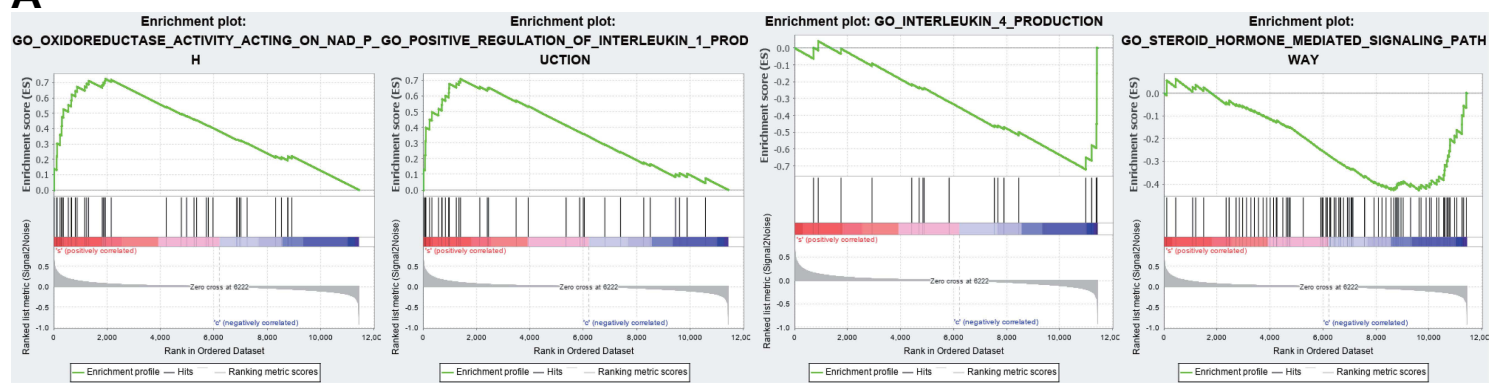

B

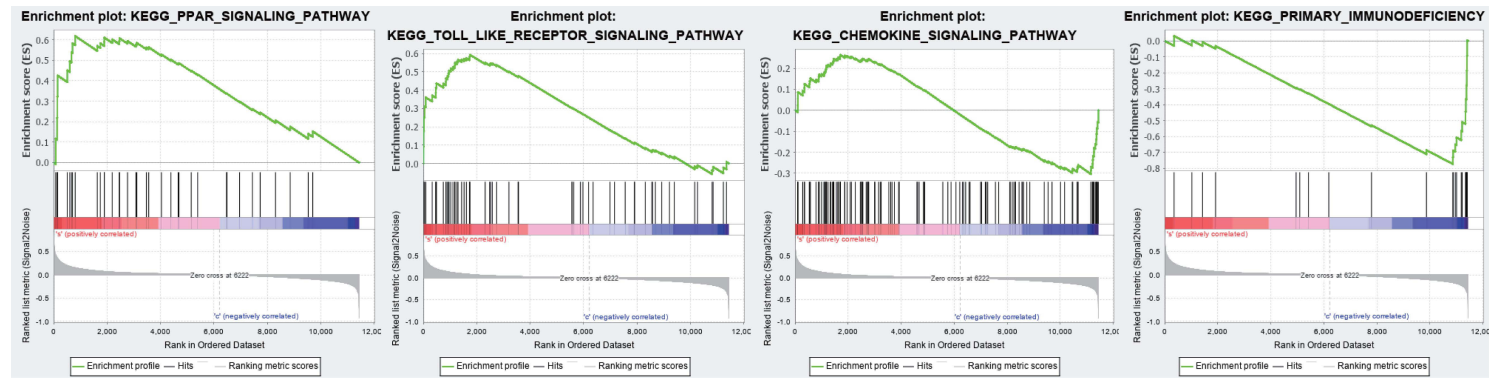

C
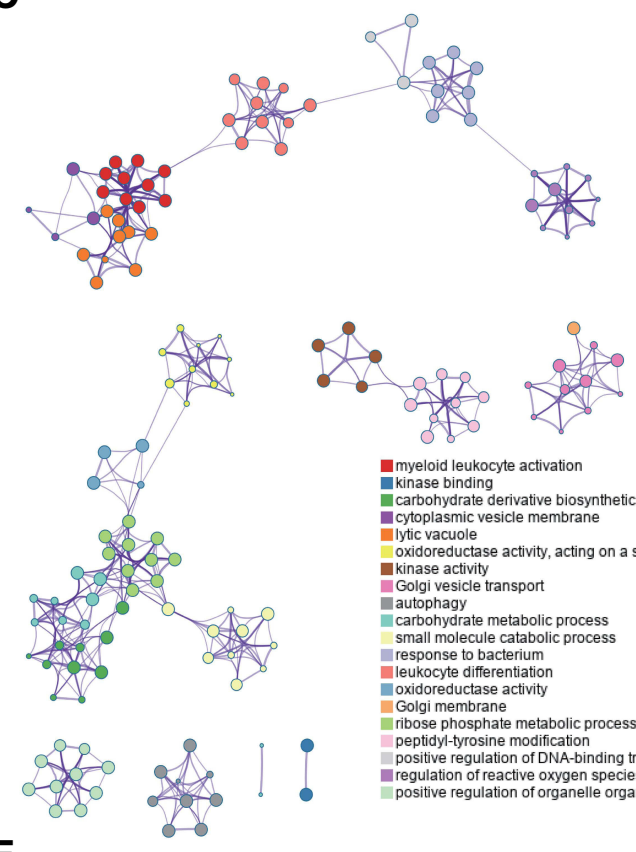

Emyeloid leukocyte activation Kinase binding
carbohydrate derivative biosynthetic process - carbohydrate derivative biosynth
cytoplasmic vesicle membrane - lytic vacuole
oxidoreductase activity, acting on a sulfur grou kinase activity Golgi vesicle transport autophagy
carbohydrate metabolic process carbohydrate metabolic process
small molecule catabolic proces response to bacterium - leukocyte differentiation oxidoreductase acti
Golgi membrane ribose phosphate metabolic proces peptidyl-tyrosine modification
positive regulation of DNA-binding transcription positve regulation of DNA-binding transcription
regulation of reactive oxygen species metabolic positive regulation of organelle organization

D

E

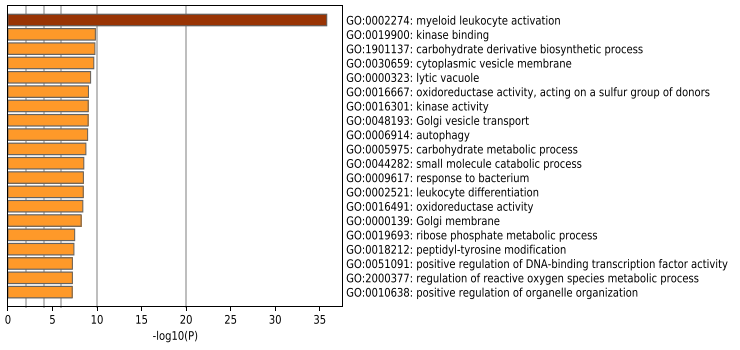

$\mathbf{F}$
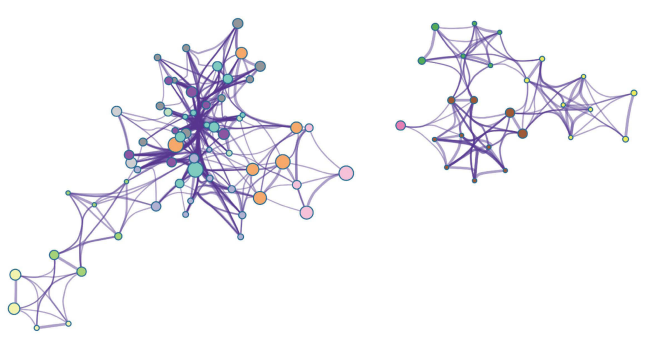

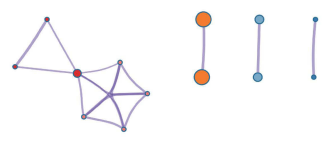

glycosaminoglycan biosynthesis - Sulfur metabolism - Amino sugar and nucleotide sugar Endocytosis
Pentose phosphate pathway, oxidative phase - Carbon metabolism HIF-1 signaling pathway
- Amyotrophic lateral sclerosis MAPK signaling pathway Alzheimer's disease
EGFR tyrosine kinase inhibitor resistance - Glycosaminoglycan biosynthesis - heparan sulfate Rap1 signain Non-alcoholic fatty liver disease (NAFLD) Cytokine-cytokine receptor interaction - Shigellosis signaling pathway Prolactin signaling pathway

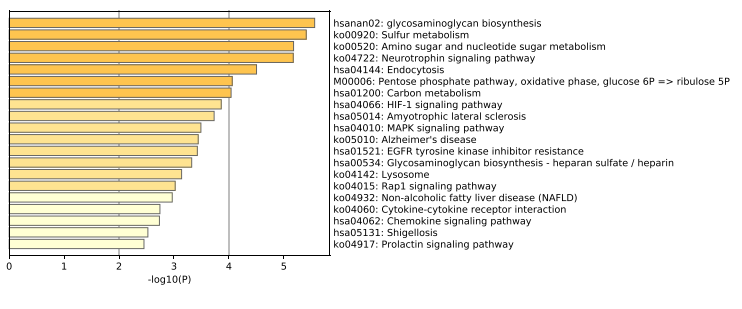

Figure 2 Gene functional enrichment analysis of the brown model genes by Gene Set Enrichment Analysis (GSEA) and Metascape. (A) Gene ontology (GO) analyses by GSEA. (B) Kyoto Encyclopedia of Genes and Genomes (KEGG) analyses by GSEA. (C) Enrichment_GO_ColorByCluster analyses by Metascape. (D) Enrichment_KEGG_ColorByCluster analyses by Metascape. Enrichment_heatmap_HeatmapSelected KEGG analyses by Metascape.

(E) Enrichment_heatmap_HeatmapSelected GO analyses by Metascape. (F) 
A

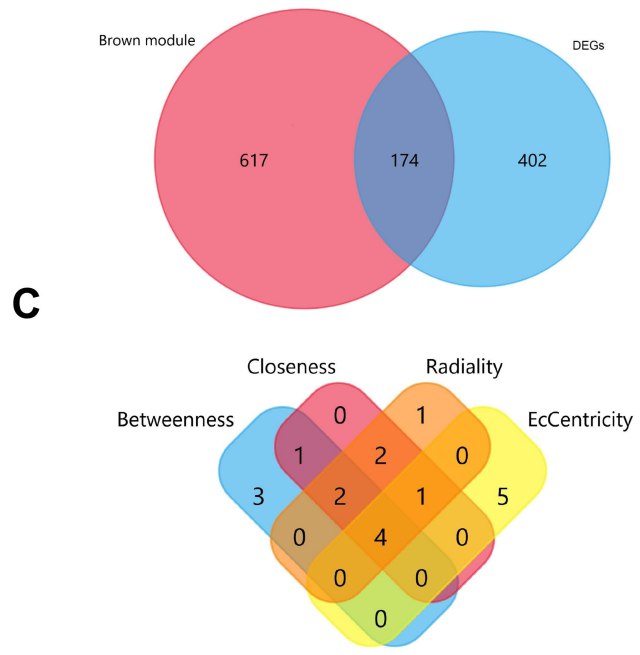

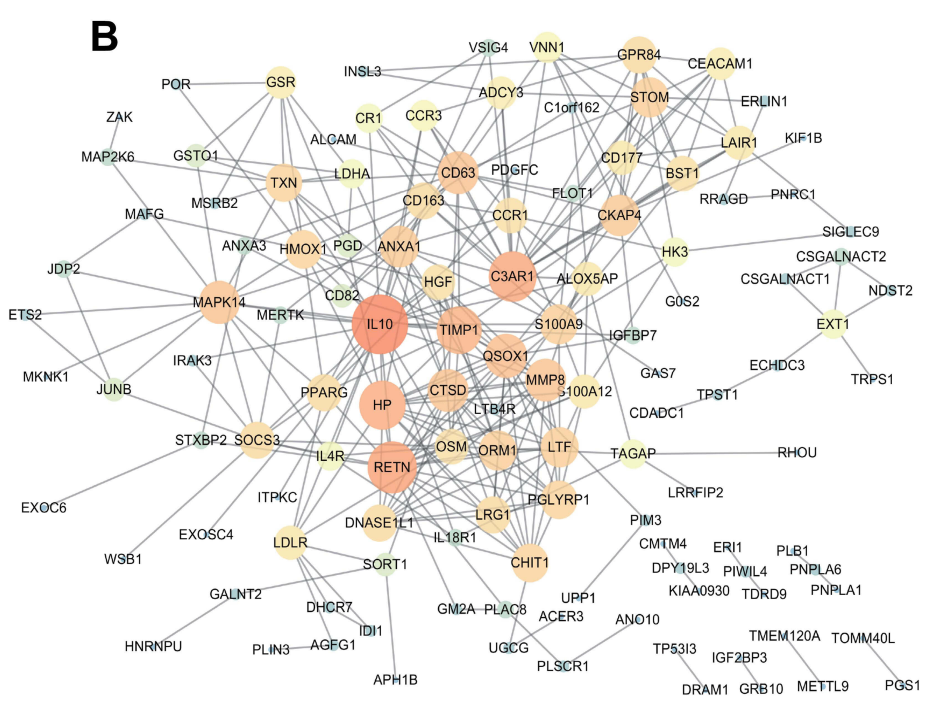

D

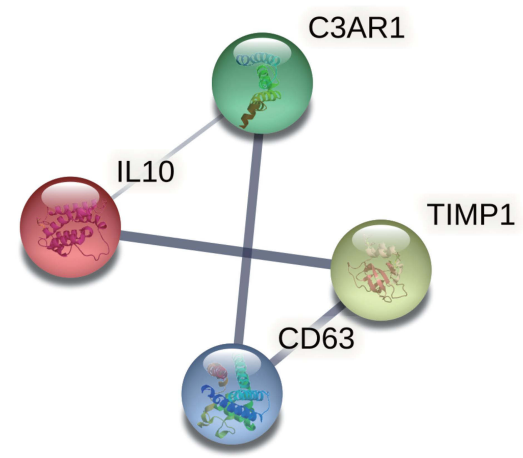

Figure 3 Relationship between differentially expressed Genes (DEGs). (A) The DEGs in the brown model genes. (B) Protein-protein interaction (PPI) network, the higher the number of connections, the larger the protein. (C) Common hub genes identified using different algorithms. (D) The common hub genes of the PPI network.

\section{Prediction and Functional Annotation Associated with Hub Genes}

GO and KEGG analyses were performed using DIANA. GO and KEGG analysis results were significantly enriched in "Transforming growth factor (TGF)-beta signaling pathway", "MAPK mitogen-activated protein kinase (MAPK) signaling pathway", "leukocyte migration", "toll-like receptor signaling pathway", and others (Figure 5).

\section{Statistical Analysis}

The ROC curves of common hub genes are shown in Figure 6.

\section{Identification of Candidate Molecule Drugs for Hub Genes}

Small molecule drugs for septic shock that could be used to target hub genes were screened using the CMap database. Small molecule drugs were filtered by the number of instances $(n>3)$ and P-values $<0.05$, as shown in Table 3.

\section{The Successful Construction of the Animal Model}

As shown in Figure 7, the relative expression levels of IL-6, TNF- $\alpha$, and lactic acid were significantly higher in the septic shock group compared with the control groups $(P<0.05$, Figure $7 \mathrm{~A}-\mathrm{C})$. 
Table 2 A Summary of Hub Genes

\begin{tabular}{|c|c|c|}
\hline Symbol & Description & Function \\
\hline ILIO & Interleukin 10 & $\begin{array}{l}\text { GO:0002875 negative regulation of chronic inflammatory response to antigenic stimulus; } \\
\text { GO:0060302 negative regulation of cytokine activity; } \\
\text { GO:1904057 negative regulation of sensory perception of pain }\end{array}$ \\
\hline C3ARI & Complement C3a receptor I & $\begin{array}{l}\text { GO:0002430 complement receptor mediated signaling pathway; } \\
\text { GO:0010759 positive regulation of macrophage chemotaxis; } \\
\text { GO:0090023 positive regulation of neutrophil chemotaxis }\end{array}$ \\
\hline TIMPI & TIMP metallopeptidase inhibitor I & $\begin{array}{l}\text { GO:0002248 connective tissue replacement involved in inflammatory response wound healing; } \\
\text { GO:0002246 wound healing involved in inflammatory response; } \\
\text { GO:005 } 1045 \text { negative regulation of membrane protein ectodomain proteolysis }\end{array}$ \\
\hline CD63 & CD63 molecule & $\begin{array}{l}\text { GO:2001046 positive regulation of integrin-mediated signaling pathway; } \\
\text { GO:0035646 endosome to melanosome transport; } \\
\text { GO:0043485 endosome to pigment granule transport }\end{array}$ \\
\hline
\end{tabular}

\section{Results of RT-qPCR and ELISA for CD63 and C3ARI}

As shown in Figure 8, the relative expression levels of CD63 were significantly higher in the septic shock group compared with the control groups, according to the PCR and ELISA results $(P<0.05$, Figure 8$)$. As shown in Figure 9 , the relative expression levels of C3AR1 were significantly lower in the septic shock group compared with the control group $(P<0.05$, Figure 9).

\section{Comparison of CD63 and C3ARI Expression in Patients with Sepsis from Those with Septic Shock}

The relative expression levels of CD63 and C3AR1 in septic shock were significantly lower than those in sepsis. $(P<0.05$, Figure 10).
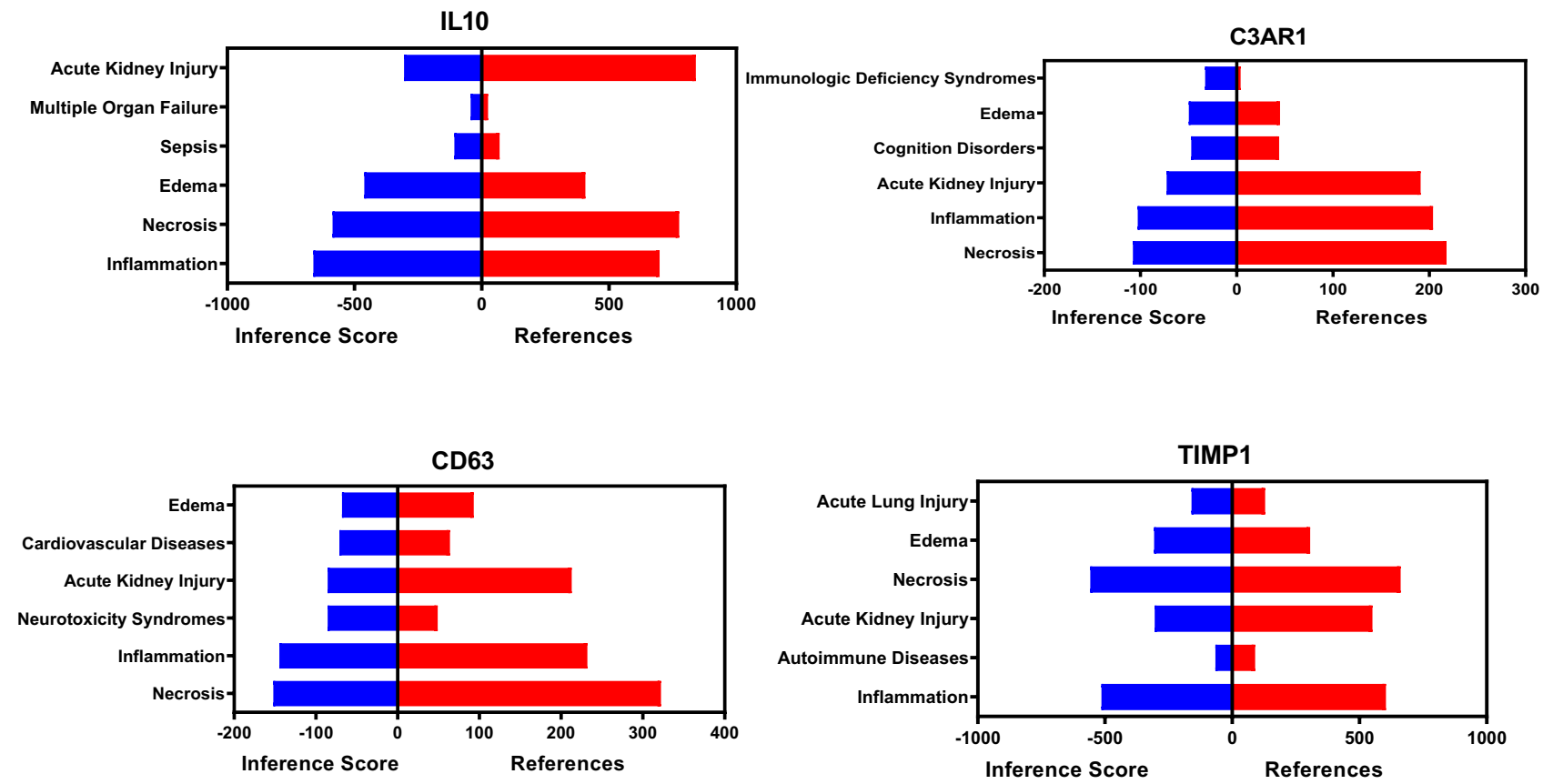

Figure 4 Relationship between the septic shock and normal groups related to hub genes based on the comparative toxicogenomics database (CTD) database. 
A

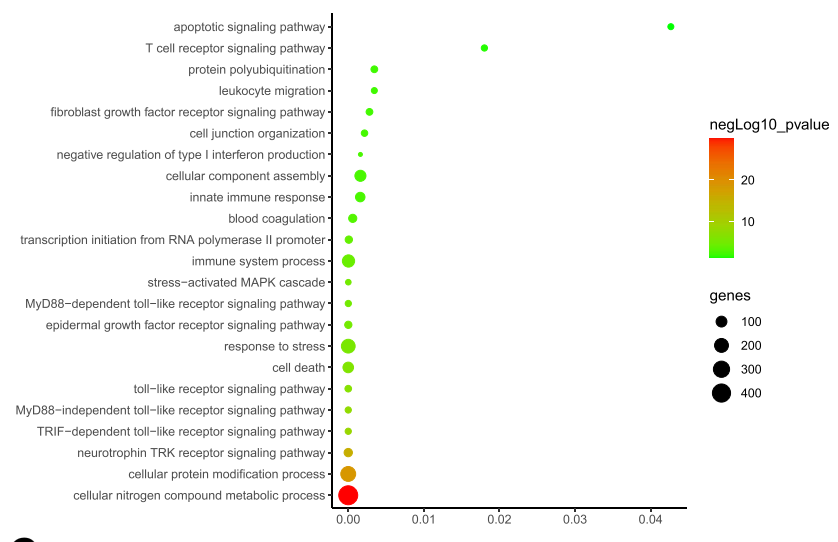

C

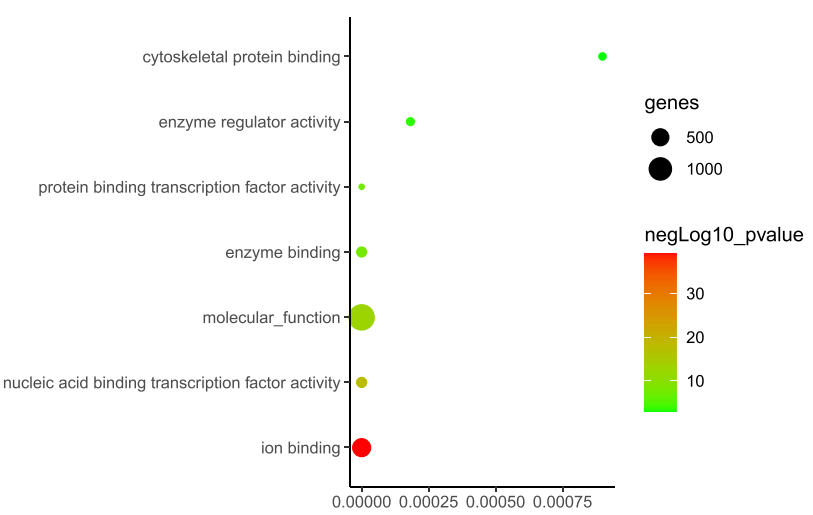

B

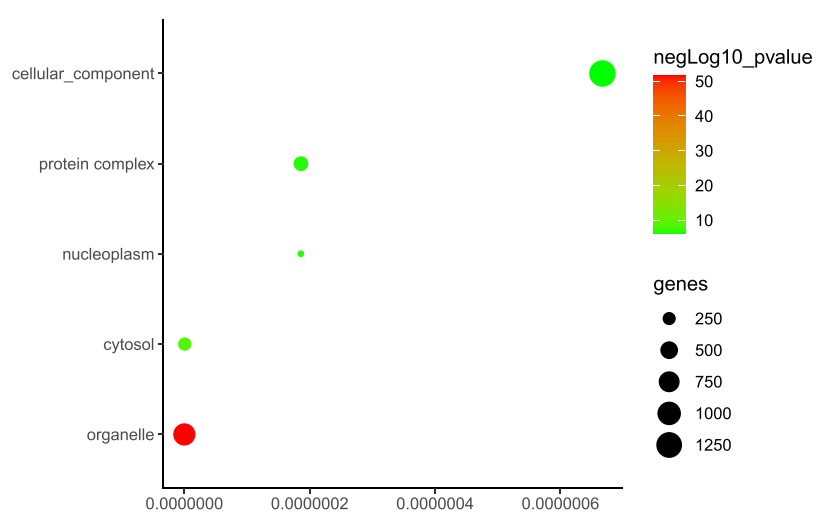

D

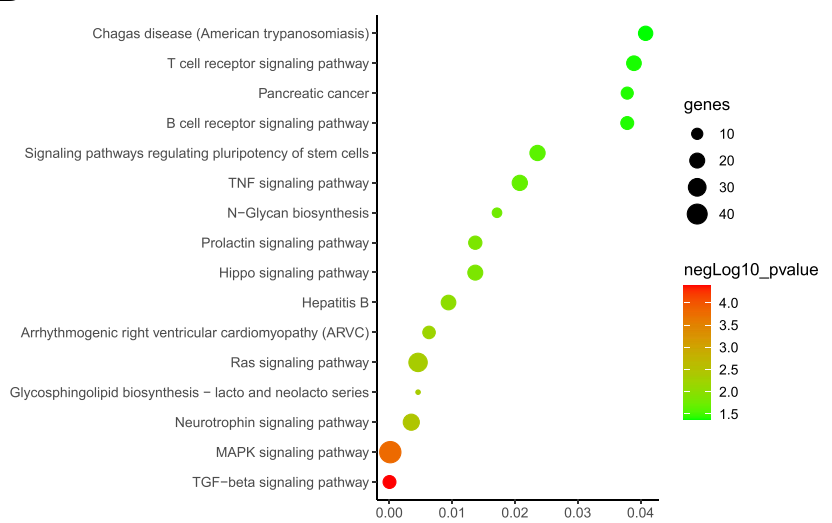

Figure 5 Functional and pathway enrichment analysis related with hub genes. (A) Biological processes (BP) analyses. (B) Cell components (CC) analyses. (C) Molecular functions (MF) analyses. (D) Kyoto Encyclopedia of Genes and Genomes (KEGG) analyses.

\section{Comparison of C5a and C5ARI Expression Between Control Mice and Septic Shock Mice}

The relative expression levels of C5a and C5AR1 in septic shock mice were significantly lower than those in control group. $(P<0.05$, Figure 11).

\section{Discussion}

Despite advances in the clinical treatment of sepsis and the discovery of new markers with diagnostic and prognostic value in recent years, septic shock remains a major challenge for intensive care units worldwide. The etiology of septic shock is still unclear, but it may be related to genetic factors, chromosomal abnormalities, gene fusion, and other factors. Therefore, it is very important to explore the molecular mechanism of septic shock to assist in the search for targeted drugs. In this study, bioinformatics techniques were used to mine microarray data related to septic shock. ${ }^{17}$ The main result of this study is that CD63 and C3AR1 were found to be significant core genes associated with septic shock. Compared with the mice in the normal control group, the expression of CD63 was significantly higher in the mice in the septic shock group, while the expression of C3AR1 was significantly lower in the septic shock group.

Previous study showed patients with sepsis showed higher expression of CD63 in platelets. ${ }^{18}$ Thrombocytopenia was associated with significantly increased CD63, CD62P, and CD31 expression and significantly down-regulated CD36 expression compared with healthy volunteers. Changes in CD63 and CD62P expression indicate platelet activation. Because CD62P, CD36, and CD31 mediate platelet interactions with leukocytes, subcutaneous matrix, and endothelial cells, as well as platelet adhesion/aggregation. This suggests that platelets are involved in leukocyte/endothelial interactions in septic shock. Studies have shown that an interleukin (IL)-1 is essential for platelet involvement in maintaining 

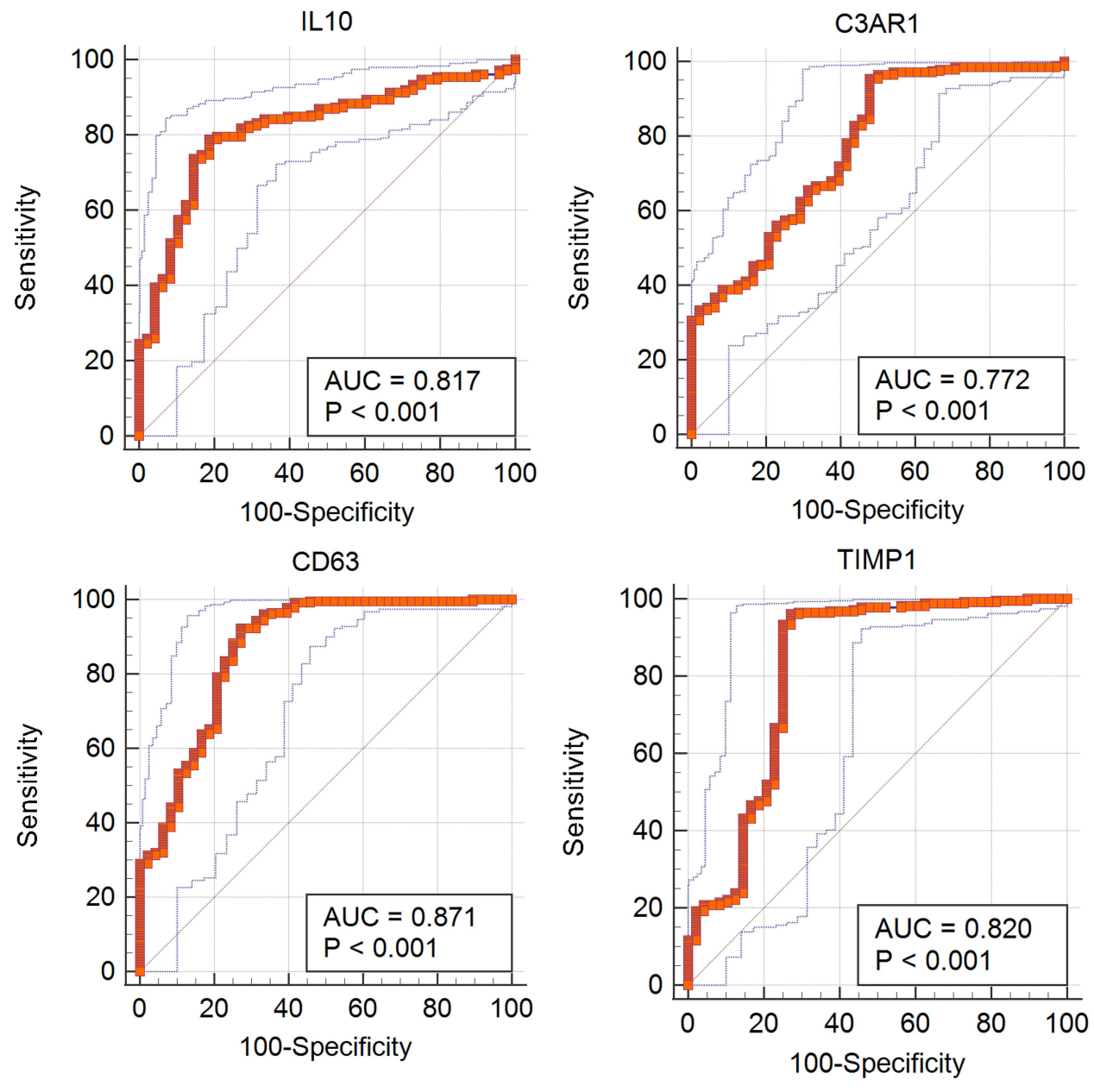

Figure 6 ROC curves of the hub genes. The orange curve represents the main line, the upper purple curve represents the upper boundary of the feasible interval, the lower purple curve represents the lower boundary of the feasible interval, and the purple line is the diagonal line.

immune homeostasis in white blood cells/endothelial cells. ${ }^{19}$ Endothelial cells may play a role in preserving microvascular patency in patients with septic shock. ${ }^{20}$

CD63 is a platelet lysosomal-intact glycoprotein, which is only slightly expressed on the surface of static platelets but is highly expressed on the surface of activated platelets. It is also one of the specific molecular markers of platelet activation. $^{21,22}$ When platelets are activated, CD63 translocates along with the "degranulation" of activated platelets. CD63 is expressed on the surface of platelet membranes and can transmit an activation signal for neutrophils that promotes the adhesion of neutrophils to endothelial cells, leading to vasculitis. ${ }^{23-25}$ The results of the present study showed that the positive expression rate of CD63 in platelets in the septic shock group was significantly higher than that

Table 3 A Summary of Connectivity Map That Regulate Hub Gene in Sepsis Shock

\begin{tabular}{|l|l|l|l|l|l|}
\hline & Cmap Name & Mean & $\mathbf{n}$ & Enrichment & P \\
\hline $\mathbf{I}$ & Monensin & 0.695 & 6 & 0.807 & 0.00008 \\
$\mathbf{2}$ & Verteporfin & -0.667 & 3 & -0.932 & 0.0005 \\
$\mathbf{3}$ & Ikarugamycin & 0.712 & 3 & 0.92 & 0.00112 \\
$\mathbf{4}$ & Tetrahydroalstonine & 0.611 & 4 & 0.829 & 0.00127 \\
$\mathbf{5}$ & Cefamandole & 0.662 & 4 & 0.791 & 0.0033 \\
$\mathbf{6}$ & Etoposide & 0.662 & 4 & 0.791 & 0.00372 \\
\hline
\end{tabular}


A

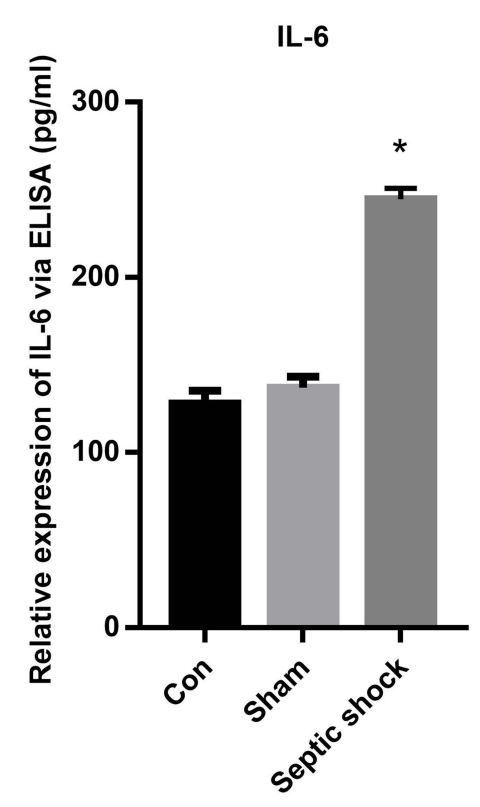

B

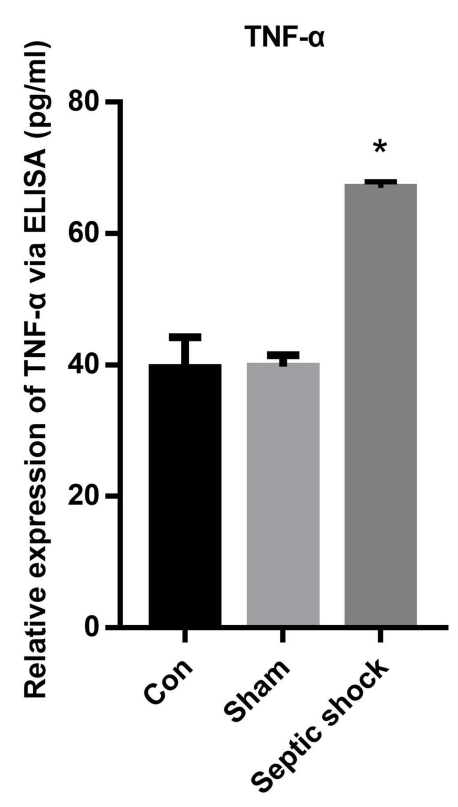

C

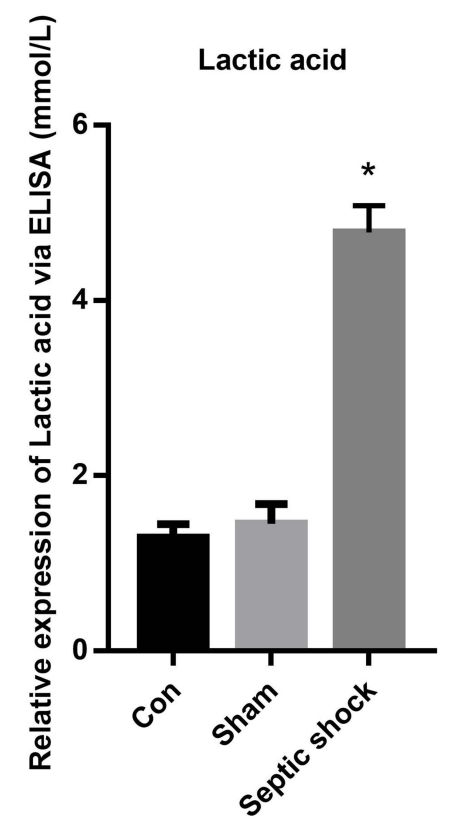

Figure 7 The construction of the animal model of septic shock. (A-C) Expression levels of interleukin-6 (IL-6), TNF- $\alpha$, and lactic acid. *P $\leq 0.05$.

A

CD 63

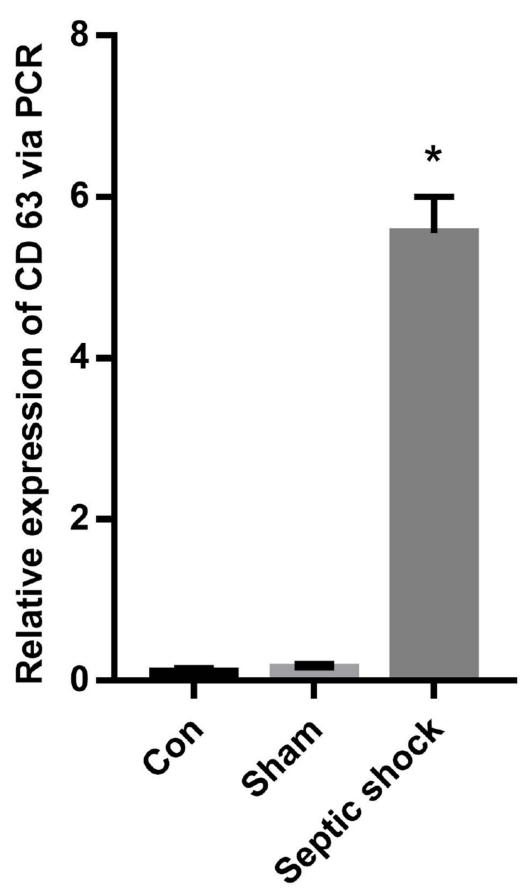

B

CD 63

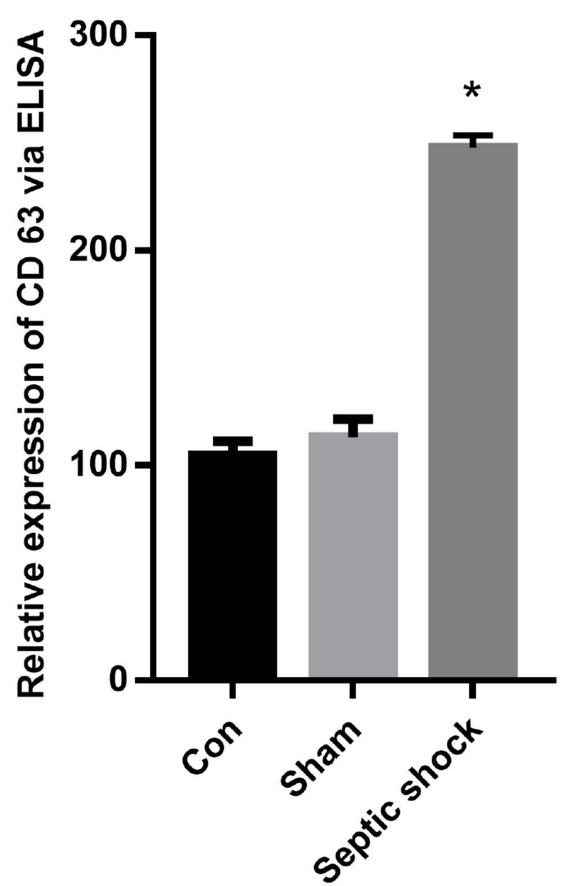

Figure 8 Results of RT-qPCR and ELISA analysis for CD63. (A) CD63 expression in control group (B) CD63 expression in septic shock group. *P $\leq 0.05$. 
A

C3AR1

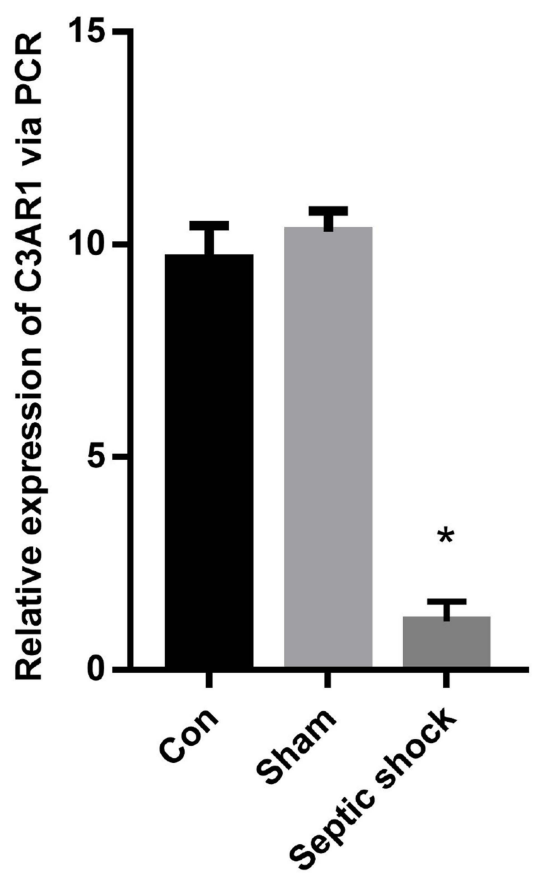

B

\section{C3AR1}

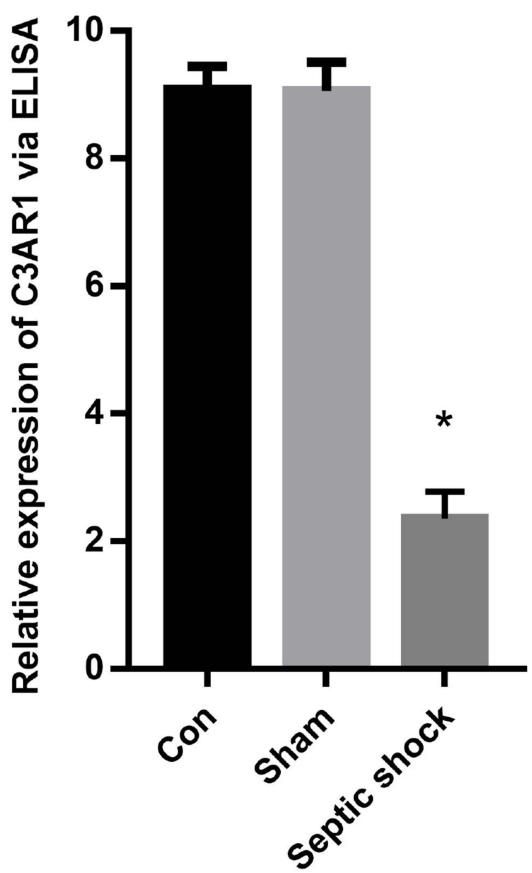

Figure 9 Results of RT-qPCR reverse transcription polymerase chain reaction and ELISA analysis for C3ARI. (A) C3ARI expression in septic shock group (B) C3ARI expression in control group. ${ }^{*} \mathrm{P} \leq 0.05$.
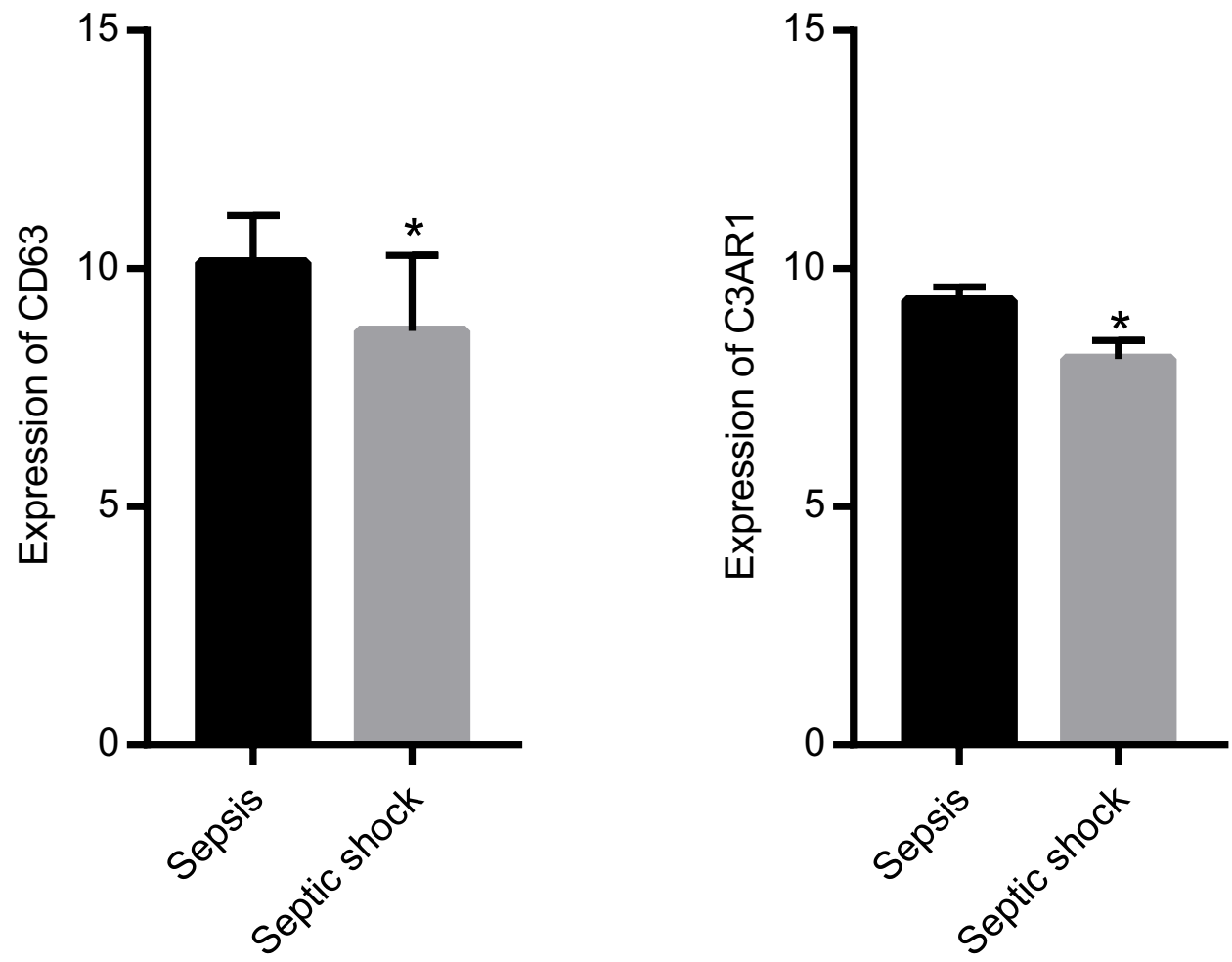

Figure 10 Comparison of $C D 63$ and C3ARI expression in patients with sepsis from those with septic shock. *P $\leq 0.05$. 

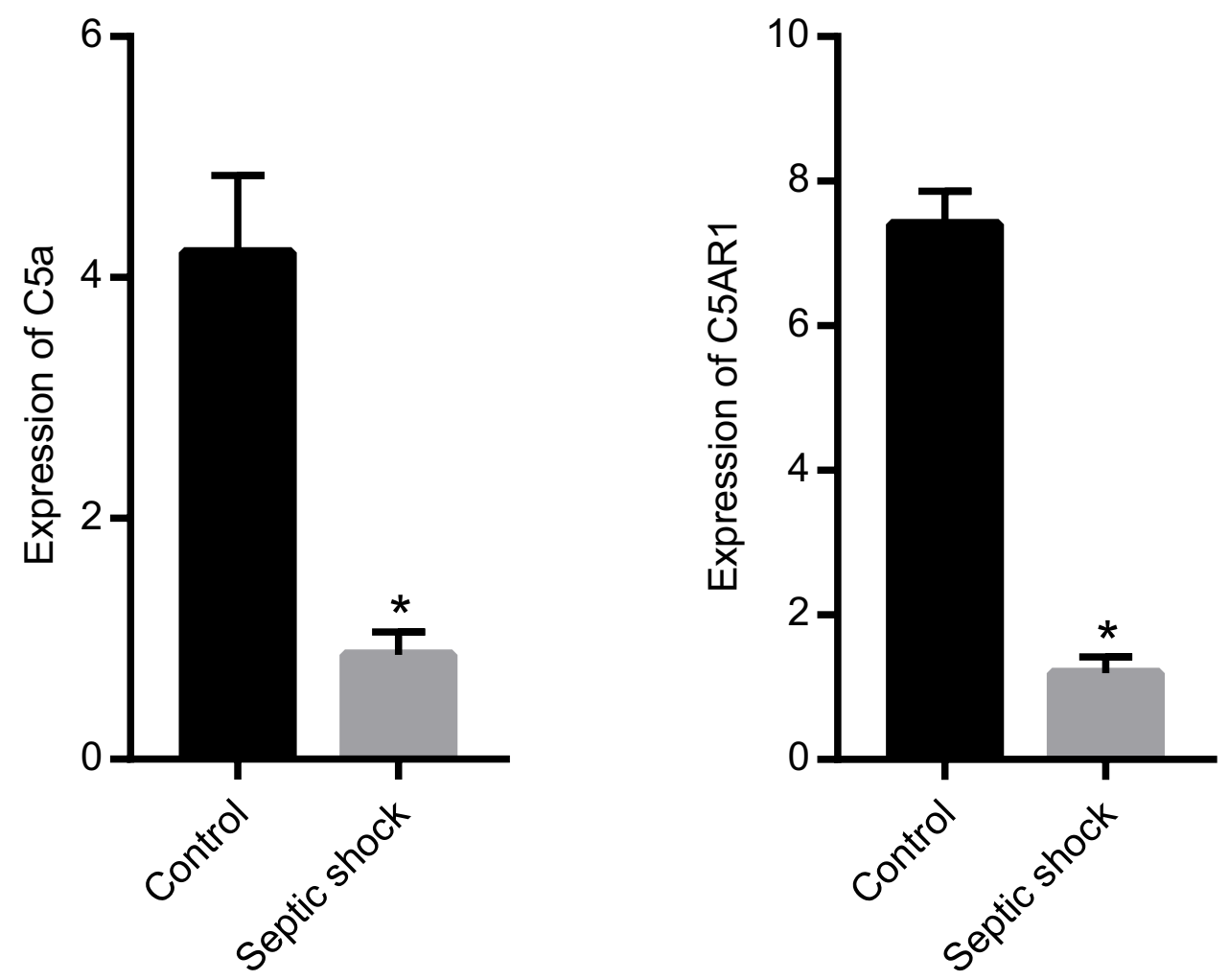

Figure II Comparison of $\mathrm{C} 5 \mathrm{a}$ and $\mathrm{C} 5 \mathrm{ARI}$ expression between control mice and septic shock mice. $* \mathrm{P} \leq 0.05$.

in the normal control group, and platelet activation increased with the risk of septic shock patients, indicating that platelet activation is an important pathological basis of sepsis. ${ }^{26}$ It is suggested that the positive expression rate of CD63 in platelets may be used as a laboratory indicator to predict the progression of septic shock. The possible mechanisms of increased platelet activity in patients with septic shock are as follows: increased capillary permeability and endothelial cell injury in patients with septic shock, on the basis of which a large number of platelet activating factors are activated; as the disease progresses, platelet activating factors interact with other pro-inflammatory cytokines, inflammatory mediators, and vasoactive substances through positive and negative feedback regulation to form a vicious cycle and further activate platelets. In addition, endotoxins and other bacterial products can activate platelets by activating monocytes and neutrophils.

CD63 is involved in the signal transduction process of various immune cell types and may be involved in the immune metabolic mechanism of cell and organ dysfunction in sepsis. ${ }^{27,28}$ Many studies have shown that CD63 is involved in the intracellular transport of pathogens such as bacteria and viruses into the endosomal system. ${ }^{29,30} \mathrm{CD} 63$ interacts directly or indirectly with many different pathogens, cells and proteins because it is not only highly expressed in the transmembrane region of exosomes, but also located in intracellular organelles. ${ }^{31,32}$ In addition, CD63 has been extensively studied as a general agent of viral infection, including human papillomavirus, human immunodeficiency virus-1, and hepatitis $\mathrm{C}$ virus. These studies suggest that exhaustion of CD63 or anti-CD63 antibody therapy can reduce the infectivity of the virus. ${ }^{33}$ And exosome CD63 may be involved in triggering infection and may influence the host immune system.

Exosome CD63 is also involved in several inflammatory processes in response to infection. In addition, CD63 is involved in monocyte adhesion, morphologic change, and proliferation, inducing various types of multinucleated giant cells to serve the first line of host defense, and triggering immune inflammatory responses to infection. ${ }^{34}$ These immune cells activated by CD63 may cause inflammatory organ damage, which can lead to multiple organ failure and ultimately death.

Compared with the normal group, C3AR1 gene expression was lower in the septic shock group. According to Napier et al RAW cells deficient of C3AR1 express lower levels of IL-6 and TNF-alpha than controls after LPS stimulation. 
Caspase-11 expression is induced by TLR4 and interferon receptor activation, and caspase-11 activation is directly stimulated by cytoplasmic LPS. C3AR is required for upregulation of caspase-11 homologous caspase- 4 and caspase- 5 in primary human macrophages during inflammation, and C3AR and Caspase-5 transcripts are highly expressed in patients with severe sepsis. C3AR is a key mediator of the CPB1-C3-C3AR signaling pathway, early produces pro-inflammatory mediators in vivo, including caspase-11, and influences the severity and outcome of the disease in the endotoxemia model of severe sepsis. LPS injection is characterized by thrombocytopenia and renal dysfunction, and glomerular endothelial p-selectin upregulation is associated with $\mathrm{C} 3$ and fibrin deposition and platelet aggregation. The functional role of $\mathrm{C} 3 \mathrm{a}$ is to significantly reduce glomerular fibrin (profibrin) and platelet clots. ${ }^{35}$

C3AR1 is a protein-coding gene. Diseases associated with C3AR1 include atypical hemolytic uremic syndrome, and complement 3 deficiency. The relevant pathway is signal transduction and peptide ligand binding receptor. The GO annotations associated with this gene include $\mathrm{G}$ protein-coupled receptor activity and complement component $\mathrm{C} 3 \mathrm{~A}$ receptor activity. An important parallel gene is complement component 5a receptor 1 (C5AR1). ${ }^{36}$ Under physiological conditions, C5a specifically binds to phospho Ser338 (CD88) by inducing the generation of polymorphonucleated neutrophils (PMNs), which in turn produce reactive oxygen species and at the same time promotes the migration, adhesion, and aggregation of PMNs, enhancing the innate immune protection offered by phagocytes. However, excessive C5A in patients with systemic inflammatory syndrome inhibits the chemotaxis of neutrophils and reduces the release of reactive oxygen species, leading to further uncontrolled infection. ${ }^{37}$ Studies have found that the massive production of C5A in the body during the early stages of sepsis leads to the down-regulation of C5A receptor expression on PMNs, resulting in a significant decrease in C5AR content on the cell surface, reaching the lowest level after $24 \mathrm{~h}$, but then slowly recovering. It is speculated that this is caused by $\mathrm{C} 5 \mathrm{~A} / \mathrm{C} 5 \mathrm{AR}$ entrapment and recycling, or by promoting the synthesis and release of inflammatory mediators to inhibit apoptosis. ${ }^{38}$ The combination of C5a and C5AR not only regulates the secretion of inflammatory factors and then regulates the function of immune cells but also participates in the regulation of the body's coagulation function. ${ }^{39}$ Prothrombin plays an important role in the regulation of C5AR-mediated complement activation. In addition, a relevant study have found that reduced C5aR expression due to excessive C5a is associated with impaired neutrophil function and poor prognosis in patients with sepsis, and that $\mathrm{C} 5 \mathrm{aR}$ expression level can serve as an early marker to predict the severity of sepsis. The findings of this literature review are consistent with our results. ${ }^{40}$ Therefore, it is speculated that the C3AR1 gene plays an important role in the development of septic shock.

Carboxypeptidase B1 (Cpb1), is required for caspase-11 gene expression and subsequent Caspase-11-dependent cell death. $\mathrm{Cpb} 1$ modifies the cleavage products of $\mathrm{C} 3$, binds to and activates $\mathrm{C} 3 \mathrm{AR}$, and then modulates innate immune signaling. The Cpb1-C3-C3AR pathway induces caspase-11 expression by amplifying MAPK activity downstream of TLR4 and Ifnar activation, and mediates LPS -induced sepsis (endotoxemia) severity and disease prognosis. C3AR is required for up-regulation of caspase- 11 homologs Caspase- 4 and 5 in primary human macrophages during inflammation, and C3AR1 and Caspase-5 transcripts are highly expressed in patients with severe sepsis. Therefore, these pathways are important in human sepsis. ${ }^{22}$

C3AR1 is thought to be limited to innate immune responses and complement cascades. However, the role of C3AR1 has been extended to involve cancer, neurogenesis and pituitary hormone release. It has been proved for the first time that down-regulation of C3AR1 expression is significantly associated with poor prognosis in patients with sepsis. ${ }^{41}$ In addition, studies have shown that the expression of C3AR1 is significantly up-regulated in patients with sepsis. ${ }^{42}$

However, there are few domestic and foreign studies on CD63, C3AR1 and septic shock related to the small molecule drugs that target central genes of septic shock screened out in this study, such as "monensin", "verteporfin", "ikarugamycin", "tetrahydroalstonine", "cefamandole" and "etoposide". This study provides a new direction for future research on septic shock.

Despite the rigorous bioinformatics analysis used in this study, there are still some deficiencies. Clinical septic shock specimens were not used to further verify the function of CD63 and C3AR1 in this study. Therefore, in future research, we should carry out an in-depth exploration of this aspect. 


\section{Conclusions}

In summary, it is particularly important to study further the molecular mechanism of septic shock. CD63 and C3AR1 are significant core genes of septic shock, which may serve as potential molecular targets for future studies of septic shock.

\section{Abbreviations}

CD63, cluster of differentiation 63; C3AR1, complement component 3a receptor 1; GSE, Gluten-Sensitive Enteropathy; GEO, Gene Expression Omnibus; DEGs, differently expressed genes; WGCNA, Weighted gene co-expression network analysis; KEGG, Kyoto Encyclopedia of Genes and Genomes; GO, gene ontology; Rap1, Ras-proximate-1; IL-6, interleukin-6; TNF- $\alpha$, Tumor Necrosis Factor- $\alpha$; ELISA, enzyme-linked immunosorbent assay; RT-qPCR, Real Time Quantitative PCR; MODS, multiple organ dysfunction syndrome; FC, Fold-changes; FDR, false discovery rate; TOM, topological overlap matrix; GSEA, Gene Set Enrichment Analysis; PPI, protein-protein interaction; 2D, twodimensional; CTD, comparative toxicogenomics database; ROC, receiver operating characteristic; Cmap, connectivity map; C57BL/6, C57 black 6; TNF, tumor necrosis factor; PCR, Polymerase Chain Reaction; RNA, RibonucleicAcid; cDNA, complementary Deoxyribonucleic acid; Taq, Thermus aquaticus; dNTP, deoxy-ribonucleoside triphosphate; PPAR, peroxisome proliferator-activated receptor; TGF, Transforming growth factor; MAPK, mitogen-activated protein kinase; C5AR1, complement component 5a receptor 1; PMNs, polymorphonucleated neutrophils; MF, molecular functions; BP, biological processes.

\section{Data Sharing Statement}

The datasets generated/analysed during the current study are available. The datasets generated/analysed during the current study are available in the corresponding author repository.

\section{Ethics Statements}

This study was approved by the Ethics Committee of the second hospital of Hebei Medical University. Written informed consent was obtained from all patients.

All experiments were approved by Animal Care and Use Committee of the Hebei Medical University. All institutional and national guidelines for the care and use of laboratory animals were followed.

\section{Author Contributions}

All authors made a significant contribution to the work reported, whether that is in the conception, study design, execution, acquisition of data, analysis and interpretation, or in all these areas; took part in drafting, revising or critically reviewing the article; gave final approval of the version to be published; have agreed on the journal to which the article has been submitted; and agree to be accountable for all aspects of the work.

\section{Funding}

There is no funding to report.

\section{Disclosure}

The authors declare that they have no competing interests.

\section{References}

1. Singer M, Deutschman CS, Seymour CW, et al. The third international consensus definitions for sepsis and septic shock (sepsis-3). JAMA. 2016;315 (8):801-810. doi:10.1001/jama.2016.0287

2. Russell JA, Rush B, Boyd J. Pathophysiology of septic shock. Crit Care Clin. 2018;34:43-61. doi:10.1016/j.ccc.2017.08.005

3. Font MD, Thyagarajan B, Khanna AK. Sepsis and septic shock - basics of diagnosis, pathophysiology and clinical decision making. Med Clin North Am. 2020;104:573-585. doi:10.1016/j.mcna.2020.02.011

4. Esposito S, De Simone G, Boccia G, De Caro F, Pagliano P. Sepsis and septic shock: new definitions, new diagnostic and therapeutic approaches. J Glob Antimicrob Resist. 2017;10:204-212. doi:10.1016/j.jgar.2017.06.013

5. Hecker A, Reichert M, Reuß CJ, et al. Intra-abdominal sepsis: new definitions and current clinical standards. Langenbecks Arch Surg. 2019;404:257-271. doi:10.1007/s00423-019-01752-7 
6. Salomão R, Ferreira BL, Salomão MC, Santos SS, Azevedo L, Brunialti M. Sepsis: evolving concepts and challenges. Braz J Med Biol Res. 2019;52:e8595. doi:10.1590/1414-431x20198595

7. Pool R, Gomez H, Kellum JA. Mechanisms of organ dysfunction in sepsis. Crit Care Clin. 2018;34(1):63-80. doi:10.1016/j.ccc.2017.08.003

8. Ziesmann MT, Marshall JC. Multiple organ dysfunction: the defining syndrome of sepsis. Surg Infect. 2018;19(2):184-190. doi:10.1089/ sur.2017.298

9. Minasyan H. Sepsis and septic shock: pathogenesis and treatment perspectives. J Crit Care. 2017;40:229-242. doi:10.1016/j.jcrc.2017.04.015

10. Vasques F, Duscio E, Romitti F, et al. Septic shock-3 vs 2: an analysis of the ALBIOS study. Crit Care. 2018;22:237. doi:10.1186/s13054-0182169-8

11. Gotts JE, Matthay MA. Sepsis: pathophysiology and clinical management. BMJ. 2016;353:i1585. doi:10.1136/bmj.i1585

12. Angarica VE, Del Sol A. Bioinformatics tools for genome-wide epigenetic research. Adv Exp Med Biol. 2017;978:489-512.

13. Weissenbach J. The rise of genomics. C R Biol. 2016;339:231-239. doi:10.1016/j.crvi.2016.05.002

14. Shendure J, Findlay GM, Snyder MW. Genomic medicine-progress, pitfalls, and promise. Cell. 2019;177:45-57. doi:10.1016/j.cell.2019.02.003

15. Qiu P, Wang L, Ni J, Zhang Y. Associations between HMGB1 gene polymorphisms and susceptibility and clinical outcomes in Chinese Han sepsis patients. Gene. 2019;687:23-29. doi:10.1016/j.gene.2018.11.027

16. Toscano MG, Ganea D, Gamero AM. Cecal ligation puncture procedure. J Vis Exp. 2011;51:e2860.

17. Zetoune FS, Ward PA. Role of complement and histones in sepsis. Front Med. 2020;7:616957. doi:10.3389/fmed.2020.616957

18. Salat A, Bodingbauer G, Boehm D, et al. Changes of platelet surface antigens in patients suffering from abdominal septic shock. Thromb Res. 1999;95(6):289-294. doi:10.1016/S0049-3848(99)00046-8

19. Beutler B, Cerami A. Cachectin and tumour necrosis factor as two sides of the same biological coin. Nature. 1986;320(6063):584-588. doi: $10.1038 / 320584 \mathrm{a} 0$

20. Kirschenbaum LA, Lopez WC, Ohrum P, Tsen A, Khazin J, Astiz ME. Effect of recombinant activated protein C and low-dose heparin on neutrophil-endothelial cell interactions in septic shock. Crit Care Med. 2006;34(8):2207-2212. doi:10.1097/01.CCM.0000229880.41513.86

21. Sartain SE, Turner NA, Moake JL. Brain microvascular endothelial cells exhibit lower activation of the alternative complement pathway than glomerular microvascular endothelial cells. J Biol Chem. 2018;293:7195-7208. doi:10.1074/jbc.RA118.002639

22. Napier BA, Brubaker SW, Sweeney TE, et al. Complement pathway amplifies caspase-11-dependent cell death and endotoxin-induced sepsis severity. J Exp Med. 2016;213(11):2365-2382. doi:10.1084/jem.20160027

23. Harder JM, Williams PA, Braine CE, et al. Complement peptide C3a receptor 1 promotes optic nerve degeneration in DBA/2J mice. J Neuroinflammation. 2020;17:336. doi:10.1186/s12974-020-02011-z

24. Fattahi F, Ward PA. Complement and sepsis-induced heart dysfunction. Mol Immunol. 2017;84:57-64. doi:10.1016/j.molimm.2016.11.012

25. Sadik CD, Miyabe Y, Sezin T, Luster AD. The critical role of C5a as an initiator of neutrophil-mediated autoimmune inflammation of the joint and skin. Semin Immunol. 2018;37:21-29. doi:10.1016/j.smim.2018.03.002

26. Tiseo G, Cavarretta E, Forniti A, et al. Interplay between Nox2 activity and platelet activation in patients with sepsis and septic shock: a prospective study. Oxid Med Cell Longev. 2020;2020:4165358. doi:10.1155/2020/4165358

27. Halova I, Draber P. Tetraspanins and transmembrane adaptor proteins as plasma membrane organizers-mast cell case. Front Cell Dev Biol. 2016;4:43. doi:10.3389/fcell.2016.00043

28. Yeung L, Hickey MJ, Wright MD. The many and varied roles of tetraspanins in immune cell recruitment and migration. Front Immunol. 2018;9:1644. doi:10.3389/fimmu.2018.01644

29. Fu E, Pan L, Xie Y, et al. Tetraspanin CD63 is a regulator of HIV-1 replication. Int J Clin Exp Pathol. 2015;8(2):1184-1198.

30. Gräßel L, Fast LA, Scheffer KD, et al. The CD63-syntenin-1 complex controls post-endocytic trafficking of oncogenic human papillomaviruses. Sci Rep. 2016;6:32337. doi:10.1038/srep32337

31. Mantegazza AR, Barrio MM, Moutel S, et al. CD63 tetraspanin slows down cell migration and translocates to the endosomal-lysosomal-MIICs route after extracellular stimuli in human immature dendritic cells. Blood. 2004;104(4):1183-1190. doi:10.1182/blood-2004-01-0104

32. Pols MS, Klumperman J. Trafficking and function of the tetraspanin CD63. Exp Cell Res. 2009;315(9):1584-1592. doi:10.1016/j.yexcr.2008.09.020

33. Park JH, Park S, Yang JS, et al. Discovery of cellular proteins required for the early steps of HCV infection using integrative genomics. PLoS One. 2013;8(4):e60333. doi:10.1371/journal.pone.0060333

34. Champion TC, Partridge LJ, Ong SM, et al. Monocyte subsets have distinct patterns of tetraspanin expression and different capacities to form multinucleate giant cells. Front Immunol. 2018;9:1247. doi:10.3389/fimmu.2018.01247

35. Morigi M, Galbusera M, Gastoldi S, et al. Alternative pathway activation of complement by Shiga toxin promotes exuberant C3a formation that triggers microvascular thrombosis. J Immunol. 2011;187(1):172-180. doi:10.4049/jimmunol.1100491

36. Herrmann JB, Muenstermann M, Strobel L, et al. Complement C5a receptor 1 exacerbates the pathophysiology of N. meningitidis sepsis and is a potential target for disease treatment. mBio. 2018;9. doi:10.1128/mBio.01755-17

37. Fattahi F, Frydrych LM, Bian G, et al. Role of complement C5a and histones in septic cardiomyopathy. Mol Immunol. 2018;102:32-41. doi:10.1016/j.molimm.2018.06.006

38. Vollrath JT, Marzi I, Herminghaus A, Lustenberger T, Relja B. Post-traumatic sepsis is associated with increased C5a and decreased TAFI levels. J Clin Med. 2020;9:1230. doi:10.3390/jcm9041230

39. Sommerfeld O, Medyukhina A, Neugebauer S, et al. Targeting complement C5a receptor 1 for the treatment of immunosuppression in sepsis. Mol Ther. 2021;29:338-346. doi:10.1016/j.ymthe.2020.09.008

40. Xu R, Lin F, Bao C, Wang FS. Mechanism of C5a-induced immunologic derangement in sepsis. Cell Mol Immunol. 2017;14:792-793. doi:10.1038/ cmi.2017.68

41. Xu C, Xu J, Lu L, et al. Identification of key genes and novel immune infiltration-associated biomarkers of sepsis. Innate Immun. 2020;26 (8):666-682. doi:10.1177/1753425920966380

42. Cai G, Zhou Y, Wen M. [Clinical importance of screening differential gene set of monocytes based on single-cell sequencing and digital polymerase chain reaction technology for early diagnosis of sepsis]. Zhonghua Wei Zhong Bing Ji Jiu Yi Xue. 2021;33(7):779-785. Chinese. doi:10.3760/cma.j. cn121430-20210607-00832 


\section{Publish your work in this journal}

The International Journal of General Medicine is an international, peer-reviewed open-access journal that focuses on general and internal medicine, pathogenesis, epidemiology, diagnosis, monitoring and treatment protocols. The journal is characterized by the rapid reporting of reviews, original research and clinical studies across all disease areas. The manuscript management system is completely online and includes a very quick and fair peer-review system, which is all easy to use. Visit http://www.dovepress.com/testimonials.php to read real quotes from published authors. 